\title{
Strong coupling effects in hybrid plexitonic systems
}

\author{
Dzmitry Melnikau $^{\mathrm{a}}$, Ruben Esteban ${ }^{\mathrm{b}}$, Alexander A. Govyadinov ${ }^{\mathrm{c}}$, Diana Savateeva ${ }^{\mathrm{a}}$, Thomas \\ Simon $^{\mathrm{d}}$, Ana Sánchez-Iglesias ${ }^{\mathrm{e}}$, Marek Grzelczak ${ }^{\mathrm{e}, \mathrm{h}}$, Mikolaj K. Schmidt ${ }^{\mathrm{a}}$, Alexander S. Urban ${ }^{\mathrm{d}}$, Luis \\ M. Liz-Marzán ${ }^{\mathrm{e}, \mathrm{h}}$, Jochen Feldmann ${ }^{\mathrm{d}}$, Javier Aizpurua ${ }^{\mathrm{a}, \mathrm{b}}$, Yury P. Rakovich*a,b,f, \\ ${ }^{a}$ Centro de Física de Materiales (MPC, CSIC-UPV/EHU), Paseo Manuel de Lardizabal, 5, \\ Donostia-San Sebastián, Spain, 20018 \\ bonostia International Physics Center (DIPC), Paseo Manuel de Lardizabal, 4, \\ Donostia-San Sebastián, Spain, 20018 \\ ${ }^{\circ}$ CIC NanoGUNE, Avenida Tolosa 76, Donostia-San Sebastián, Spain, 20018 \\ dLudwig-Maximilians-Universität München, Amalienstrasse 54, Munich, Germany, 80799 \\ ${ }^{e}$ CIC biomaGUNE, Donostia-San Sebastián, Spain \\ ${ }^{f}$ National Research Nuclear University MEPhI (Moscow Engineering Physics Institute), Kashirskoye \\ sh., 31, Moscow, Russia, 115409 \\ ${ }^{\mathrm{h}}$ IKERBASQUE, Basque Foundation for Science, Bilbao, Spain
}

\begin{abstract}
We investigated the interactions between localized plasmons in gold nanorods and excitons in J-aggregates and were able to track an anticrossing behavior of the hybridized modes both in the extinction and in the photoluminescence spectra of this hybrid system. We identified the nonlinear optical behavior of this system by transient absorption spectroscopy. Finally using magnetic circular dichroism spectroscopy we showed that nonmagnetic organic molecules exhibit magnetooptical response due to binding to a plasmonic nanoparticles. In our experiments we also studied the effect of detuning as well as the effect of off- and on resonance excitation on the hybrid states.
\end{abstract}

Keywords: excitons, plasmons, plexitons, strong coupling, Rabi splitting, nonlinear effects, magneto-optical effect

\section{INTRODUCTION}

One of the main objectives of nanophotonics research is to control the light-matter interaction on the nanoscale. Phenomenon of plasmon-exciton resonance coupling is of great importance for this research field in both the fundamental aspects and many practical applications. Especially attractive for these applications is so called strong coupling regime when the rate of periodic and coherent energy exchange between the excitonic and plasmonic systems exceeds the rate of the losses in the system. This periodic energy exchange has an analogy with two coupled oscillators where new eigenmodes of the system arise, manifesting itself in the appearance of a double-peaked feature in the extinction spectra ${ }^{1}$. The spectral distance between these two peaks defines the value of the energy of Rabi splitting ( $\Omega R$ ) which is one of fundamental quantum-optics phenomena ${ }^{2}$. One of the advantages of plasmonic structures is the extremely low modal volume associated with their electromagnetic resonances, one of the prerequisites for achieving strong coupling strengths. However, because of the very fast decay rates of plasmons, it still remains very challenging to achieve strong coupling $\hbar$ $\Omega_{R}>0$ for single molecules or quantum dots with moderate oscillator strengths. In this respect, coupling the plasmons with excitons of organic dyes in the J-aggregated state is of significant interest for the development of advanced photonic technologies based on organic - inorganic hybrid nanosystems. This is due to the ability of J-aggregates to delocalize and migrate excitonic energy over a large number of aggregated dye molecules, which results in extremely high oscillator strength $^{3}$.

While observation of Rabi splitting in extinction or transmission spectra of exciton-plasmon (plexiton) hybrid systems has been reported in a number of publications ${ }^{4}$, photoluminescence (PL) properties of these systems in the strong coupling regime are more elusive to experimental investigation ${ }^{4,5}$. Also despite several studies in recent years showing the formation

Third International Conference on Applications of Optics and Photonics, edited by Manuel F. M. Costa, Proc. of SPIE Vol. 10453, 104532E · C) 2017 SPIE · CCC code: 0277-786X/17/\$18 · doi: 10.1117/12.2276063 
of plexcitonic states, their nature, especially at very short times, is still insufficiently investigated ${ }^{6}$. Finally, nothing is known to date about magneto-optical properties of hybrid plexitonic systems in strong coupling regime.

In this paper we present and discuss our recent results on study of strong coupling effects in (i) PL spectra of plexitonic systems, (ii) optical nonlinearities in hybrid structures and (iii) magneto-optical activity in coupled plasmon-exciton complexes.

\section{RESULTS AND DISCUSSION}

\subsection{Rabi splitting in absorption and photoluminescence spectra of plexitonic systems}

In this part we discuss our recent progress in study of optical properties of plexitonic system formed by gold nanorods and J-aggregates in strong coupling regime.

Extiction spectra of bare gold nanorods show two bands: the transverse modes (TM), related to the electron oscillations perpendicular to the long nanorod axis, and the longitudinal modes (LM), caused by the oscillations along the long nanorod axis. The spectral positions of resonances of both mode types can be controlled by the nanorods aspect ratio (Figure 1a). By using simple methods described in our previous works we created highly stable hybrid structures of these nanorods and cyanine dye JC1 in J-aggregated state ${ }^{4}$.

One of the main results of our study is presented in Figure 1b,d,e. In resonant regime (matching spectral positions of plasmonic and excitonic resonances) in the extinction spectrum of the plexitonic system the pronounced dip at $590 \mathrm{~nm}$ appears as a result of strong coupling of the excited states of J-aggregates and plasmon modes of the nanorods. Also left panel in Figure 1 demonstrates that the separation between upper resonance (UR) and lower resonance (LR) grows with the detuning of extinction bands of pure gold nanorods with different aspect ratios from the excitonic resonance of $\mathrm{J}$ aggregates. This separation reaches $710 \mathrm{meV}$ for the biggest detuning achieved in our experiments $(681 \mathrm{meV})$.
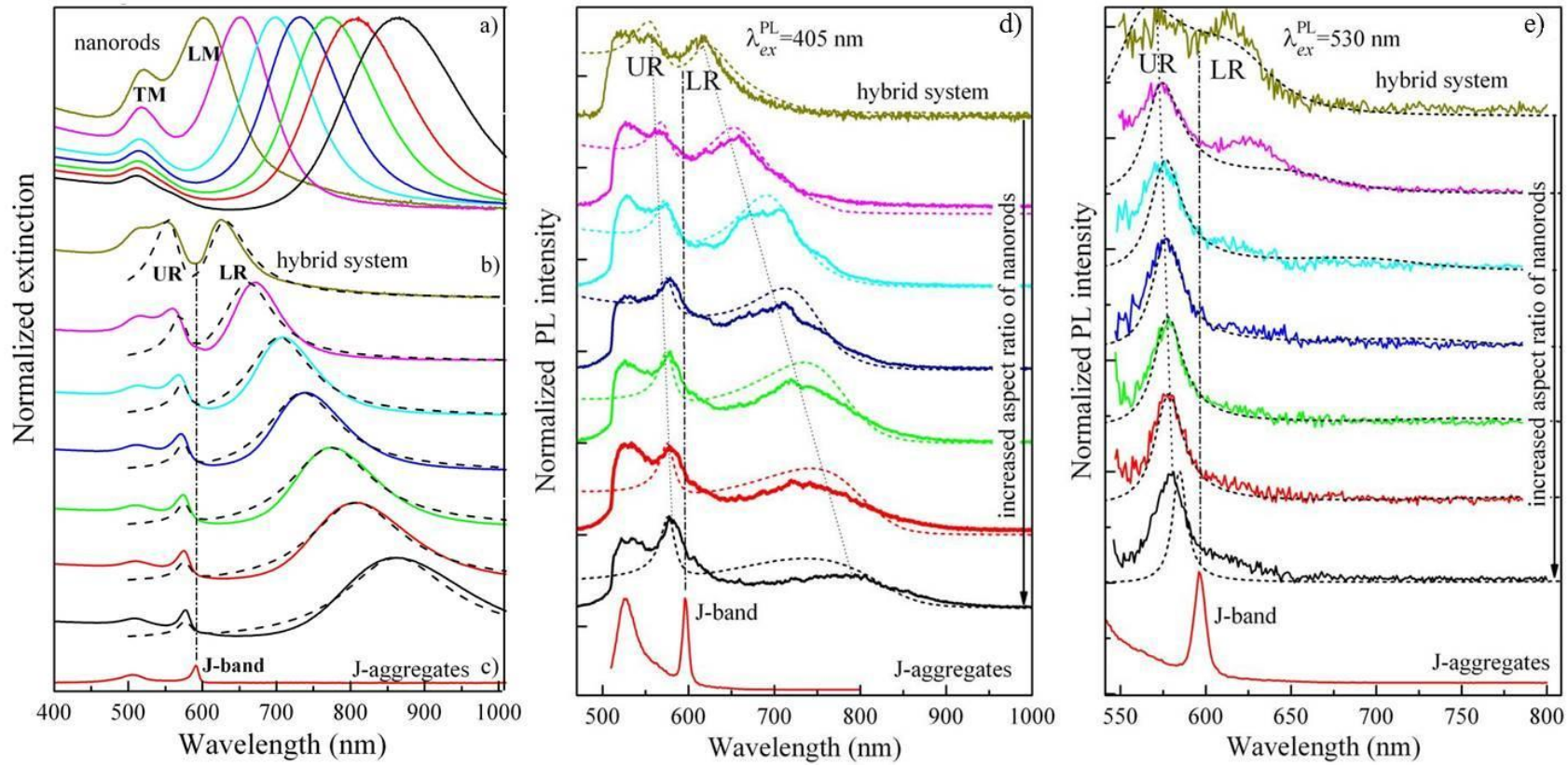

Figure 1 Left panel: Extinction spectra of gold nanorods of increased aspect ratio (from left to right) (a), hybrid plexitonic complexes formed by J-aggregates and gold nanorods of increased aspect ratio (from top to bottom) (b) and pure Jaggregates (c). Central and right panels: Normalized PL spectra of the plexitonic system formed by nanorods of different aspect ratios and J-aggregates, measured when using excitation at 405 (d) and $530 \mathrm{~nm}$ (e). Dashed curves are the normalized results of the theoretical calculations. Vertical dashed-dotted line indicates the spectral position of the J-band.

We estimated value of the Rabi splitting $\left(\Omega_{\mathrm{R}}>200 \mathrm{meV}\right)$ at zero detuning from the anticrossing behavior of the bands in extinction spectra. This value is similar to the largest splittings observed in planar organic microcavities or in plexitonic systems based on gold particles ${ }^{4}$. Next, we focused on the signature of the strong coupling in the evolution of the PL modes, which we can identify due to the pronounced emission from both the UR and LR branches. The evolution of the 
modes is consistent with the results from the extinction, which corroborates the interpretation of the modes in terms of an anti-crossing. Both the extinction and PL results are in good agreement with the results of theoretical calculations performed using a model of two interacting modes with a ratio between the coupling strength and the plasmonic losses close to 0.4 , indicative of the strong coupling regime. We also used a theoretical model of emission to analyze lineshapes of the PL spectra.

It turned out that the evolution of the spectra with increasing detuning depends on the excitation wavelength (Figure 1d,e). Under excitation at both 405 and $530 \mathrm{~nm}$ the contribution from the UR at larger energies appears in all spectra, becoming more clear as the detuning grows. However for $530 \mathrm{~nm}$ excitation (Figure 1e), the lower energy LR peak vanishes very rapidly with increasing detuning. For excitation at $405 \mathrm{~nm}$ (Figure 1d), a contribution from the two branches is observed for all detunings, and the shift of the LR peak, when comparing the two rods of smaller aspect ratio, is larger than for illumination at $530 \mathrm{~nm}$.

We explain the difference as a consequence of predominantly pumping the plasmonic mode for the smaller wavelength of PL excitation light, or the J-aggregates electronic transitions for the larger excitation wavelength. When the nanorod resonance and the J-band of J-aggregates appear at a similar energy (low detuning), both hybrid modes can be excited incoherently via either the decay processes in the metal for $405 \mathrm{~nm}$ excitation or nonradiative recombination in the Jaggregates when exciting at $530 \mathrm{~nm}$. For large detuning, one of the hybrid modes is essentially plasmon-like and the other is exciton-like, and the excitation strongly affects their relative weight, with $530 \mathrm{~nm}$ light exciting preferentially the latter and $405 \mathrm{~nm}$ a more mixed balance.

\subsection{Optical nonlinearities of plexitonic hybrid system}

According to the hybridization model UR and LR modes are result of the formation of half-plasmon, half-exciton states with 50/50 contribution from constituent parts. However our results on dependence of PL lineshape on excitation wavelength clearly indicate that this might not be the case. To get deeper inside in intrinsic character of the hybrid states we applied femtosecond transient absorption (TA) (nonlinear) spectroscopy using off- and on resonance excitation.
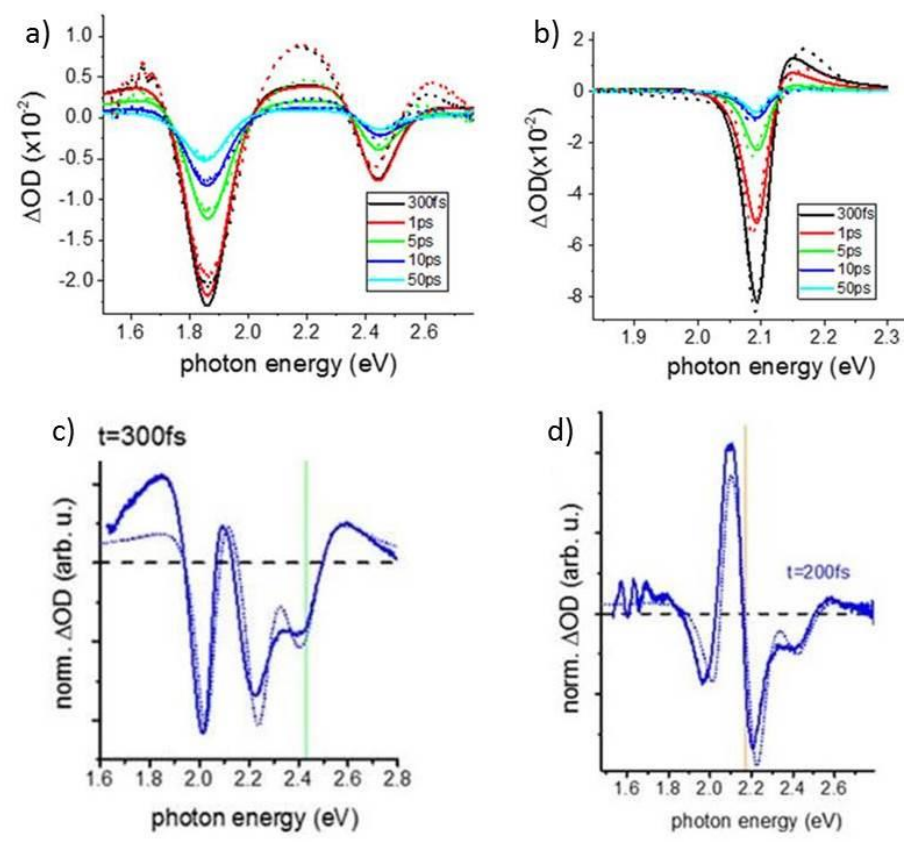

Figure 2 (a) Transient absorption spectra for bare Au nanorods: Experimental data (solid curves) and theoretical simulation (dotted curves) at different times after excitation. (b) TA spectra of J-aggregated dye molecules. (c) Experimental and modeled TA spectra for plexitonic hybrid particles excited at $2.44 \mathrm{eV}(510 \mathrm{~nm})$. (d) TA spectra for plexitonic hybrid particles excited at $2.18 \mathrm{eV}(570 \mathrm{~nm})$.

Two minima can be seen in the spectra acquired from bare Au nanorods and are located at the resonance positions of the transverse and longitudinal plasmon peaks in extinction spectra (Figure 2a). Both are merged into regions with $\Delta \mathrm{OD}>0$. 
To model these spectra we considered the LM and TM plasmon resonances as Lorentz oscillators with line shapes according to $O D(E)=\frac{f \Gamma / 2}{\left(E_{0}-E\right)^{2}+(\Gamma / 2)^{2}}$. Upon non-linear excitation three possible changes ("non-linearities") can occur: they can change their i) amplitude $f$ (bleaching if $f_{o n}<f_{o f f}$, induced absorption if $f_{o n}>f_{o f f}$ ), ii) central energy position $E_{0}$ and iii) spectral width $\Gamma$. For the bare nanorods the TA spectra can be modeled by predominantly increasing the damping of both plasmon resonances. TA spectra of the J-aggregates yield a different picture (Figure $2 \mathrm{~b}$ ). Two peaks are visible, one located at the spectral positon of the J-band around $2.1 \mathrm{eV}$ with $\Delta \mathrm{OD}<0$, and one peak with $\Delta \mathrm{OD}>0$ located between 2.125 and $2.25 \mathrm{eV}$. The latter peak is due to a cascaded biexcitonic transition, causing photoinduced absorption at higher energies. The other peak is a result of the bleaching of the J-band due to the creation of excitons by the pump pulse.

In the case of coupled plexitonic system we first investigated TA spectra at non-resonant excitation i.e. exciting the plasmon only, by tuning the pump laser to be resonant with uncoupled transverse plasmon of the nanorods. Three distinct minima are visible in spectrum, all of which reach their maximum signal for a delay time of 300 fs. (Figure 2c) The positions of these minima correspond to the LR, UR, and transverse plasmon resonances previously seen in the linear extinction spectra (Figure 1a,b). Again, experimental TA spectra can be reproduced mainly by spectral broadening of all three resonances (dotted curves).

Upon direct excitation of the hybrid plexcitonic state with pump laser tuned to $2.18 \mathrm{eV}$ ( $570 \mathrm{~nm}$ ) (to be resonant with UR) the nonlinearities are very different for short delay times. In the transient absorption spectrum acquired at 200 fs a strong peak with $\Delta \mathrm{OD}>0$ appears between 2.0 and $2.2 \mathrm{eV}$ (Figure $2 \mathrm{~d}$ ). This result shows that along with broadening an additional nonlinearity comes into play on ultrashort time scales. It turned out that a considerable spectral shift of LR and UR (narrowing of the energy splitting) is necessary to reproduce experimental spectra. This indicate that reduction of the coupling strength between the plasmon and the J-aggregate is responsible for the described phenomenon. In resonant excitation regime the direct excitation of the excitonic part of hybrid system reduces the number of excitons, interacting with the local electric field of the plasmons and the Rabi splitting diminishes within the lifetime of the exciton population.

\subsection{Magneto-optical activity of plexitonic system in strong coupling regime}

Methods based on magneto-optical (MO) activity measurements, such as magnetic circular dichroism (MCD) spectroscopy and magneto-optic Kerr effect (MOKE), take an important place in the arsenal of tools for materials identification and characterization. In this part we investigated how magneto-optical properties of a hybrid system can be affected by strong exciton-plasmon coupling.

To obtain plasmon nanoparticles with pronounced resonances at the characteristic energies of J-aggregates we selected core-shell Au@Ag nanorods of different aspect ratios. Like pure Au nanorods, Au @ Ag nanoparticles show two bands in extinction spectra: the TM and LM resonances, the spectral positions of which can be controlled by the nanorods aspect ratio. (Figure 3a, lower panel)

Figure $3 \mathrm{~b}$ shows the MCD spectra (the difference between the absorbance of left- and right-hand polarized light, $\Delta \mathrm{A}(\lambda)$ ) measured on bare nanorods of different aspect ratios under the applied magnetic field of $\pm 1 \mathrm{~T}$. As expected, we registered strong signals of magnetic circular dichroism at the TM and LM resonances of nanorods for both polarities of the magnetic field. The optical response of the hybrid plexitonic system at short wavelengths $(300-500 \mathrm{~nm}$, Figure $3 \mathrm{~b}$, lower panel), where the TM resonances of nanorods are located, is nearly unaffected by the presence of J-aggregates. In contrast, at long wavelengths $(550-800 \mathrm{~nm}$ ) we observed two extinction peaks for each nanorods aspect ratio (one at $557-575 \mathrm{~nm}$ and the other at $615-794 \mathrm{~nm}$, Figure 3b), which we attributed to UR and LR of strongly coupled plexitonic system, respectively.

The most interesting behavior, however, is exhibited by the plexitonic system in the MO response. For the highest aspect ratio (4.5) we only observe a pronounced MCD within the LR absorption band and an insignificant MCD in the UR band. This is expected because J-aggregates, as well as other organic materials, are known to exhibit no significant MO activity. Yet remarkably, for smaller aspect ratios we detect a strong MCD signal at the UR (aspect ratios from 2.5 to 4 in Figure $3 b)$. The reversal of the sign of MCD by changing the polarity of the magnetic field confirms the magnetic nature of the detected signal. 
We explain the origin of strong MO activity in J-aggregates as following. The strong coupling leads to the formation of two hybrid plexciton states, each exhibiting both excitonic and plasmonic properties. Thus, the exciton effectively acquires the MO activity from the plasmon, yielding strong MCD upon tuning of plasmonic and excitonic resonances?
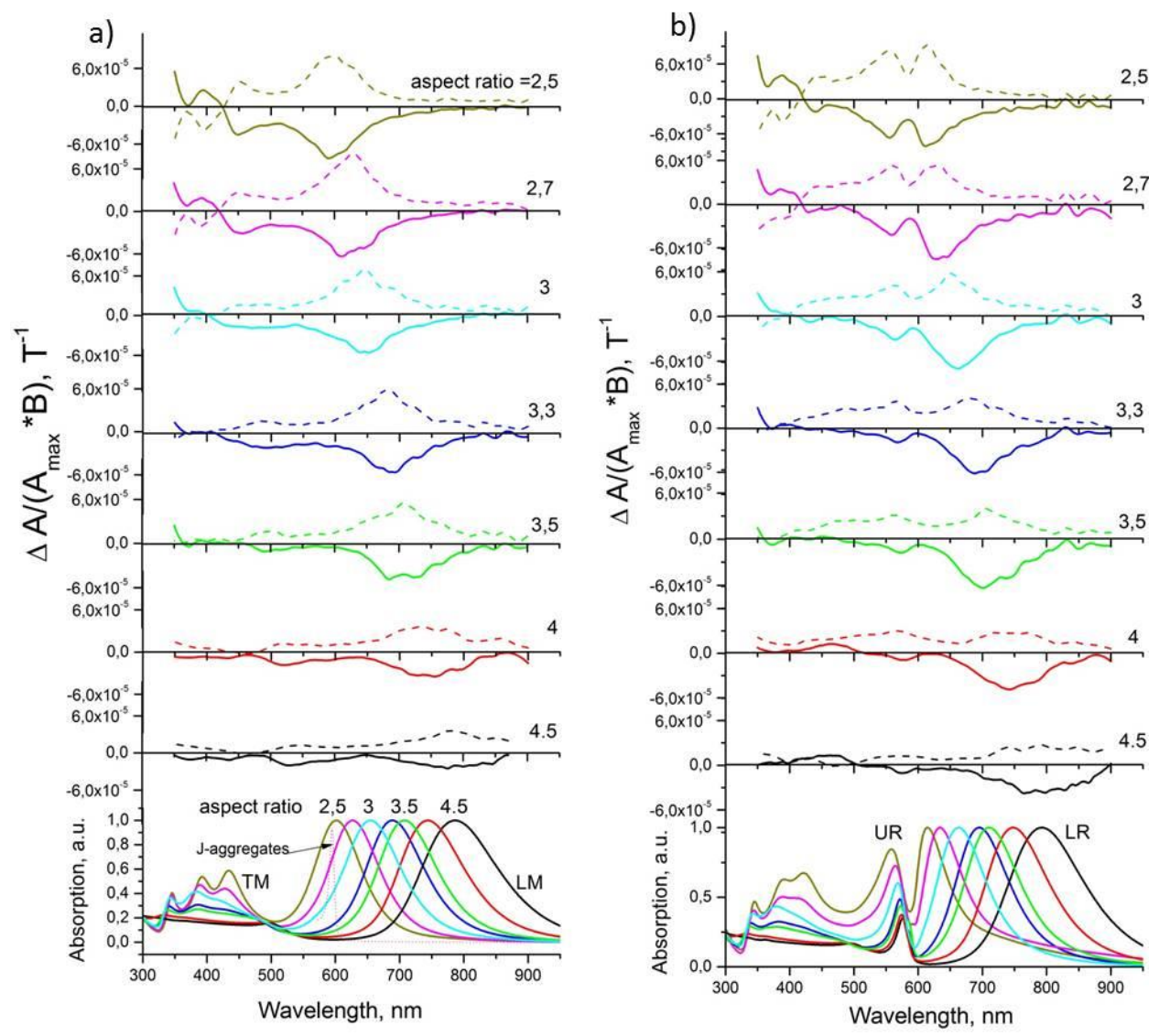

Figure 3 (a) Magnetic circular dichroism spectra, $\Delta \mathrm{A}$, (defined as the difference between absorbance for left- and right-hand circularly polarized light in the presence of a magnetic field) for bare core-shell Au@ Ag nanorods of different aspect ratios (b) MCD spectra of the hybrid system of J-aggregates and nanorods for different aspect ratios. Lower panel shows absorption spectra of the hybrid system of J-aggregates and nanorods.

\section{CONCLUSIONS}

We were able to observe pronounced Rabi splitting and track a clear anticrossing behavior of the hybridized modes in plexitonic system formed on gold nanorods coated with J-aggregated cyanine molecules not only in the extinction but also in the photoluminescence spectra. Notably, while previous studies often found the PL signal to be dominated by a single mode (emission from so-called lower polariton branch), here we followed the evolution of the two PL peaks as the plasmon energy is detuned from the excitonic resonance. The evolution of the PL line shape as the plasmon is detuned depends on the excitation wavelength, which we attribute to an incoherent excitation given by decay processes in either the metallic rods or the J-aggregates.

We identified the nonlinear optical behavior of plexcitons at short times by transient absorption spectroscopy and a simple Lorentz oscillator model. We controlled the spectral overlap of the two resonances and analyzed the effect of off- and on resonance excitation on the hybrid states.

We demonstrated that the $\mathrm{MO}$ activity of an organic compound itself can be greatly enhanced by coupling to a resonant plasmon nanoparticles. 


\section{ACKNOWLEDGEMENTS}

We acknowledge financial support from Project Fis2016.80174-P (PLASMOQUANTA) from MINECO (Ministerio de Economía y Competitividad). L.L.-M. acknowledges funding from the European Research Council (ERC Advanced Grant 267867, Plasmaquo). This study was supported by the Ministry of Education and Science of the Russian Federation, grant no. 14.Y26.31.0011.

\section{REFERENCES}

[1] Novotny L., "Strong coupling, energy splitting, and level crossings: A classical perspective," Am. J. Phys. 78(11), 1199-1202 (2010).

[2] Dicke R.H., "Coherence in Spontaneous Radiation Processes," Phys. Rev. 93, 99-110 (1954).

[3] Kobayashi, T., [J-aggregates], World Scientific, Singapore (1996).

[4] Melnikau, D.; Esteban, R.; Savateeva, D.; Sánchez-Iglesias, A.; Grzelczak, M.; Schmidt, M. K.; Liz-Marzán, L. M.; Aizpurua, J.; Rakovich, Y. P., "Rabi Splitting in Photoluminescence Spectra of Hybrid Systems of Gold Nanorods and J-Aggregates," J. Phys. Chem. Lett. 7(2), 354-362 (2016).

[5] Wersäll, M.; Cuadra, J.; Antosiewicz, T. J.; Balci, S.; Shegai, T., "Observation of Mode Splitting in Photoluminescence of Individual Plasmonic Nanoparticles Strongly Coupled to Molecular Excitons," Nano Lett. 17(1), 551-558 (2016).

[6] Simon, T.; Melnikau, D.; Sánchez-Iglesias, A.; Grzelczak, M.; Liz-Marzán, L. M.; Rakovich, Y.; Feldmann, J.; Urban, A. S., "Exploring the Optical Nonlinearities of Plasmon-Exciton Hybrid Resonances in Coupled Colloidal Nanostructures," J. Phys. Chem. C 120(22), 12226-12233 (2016).

[7] Melnikau, D.; Govyadinov, A. A.; Sánchez-Iglesias, A.; Grzelczak, M.; Liz-Marzán, L. M.; Rakovich, Y. P., "Strong magneto-Optical Response of Nonmagnetic Organic Materials Coupled to Plasmonic Nanostructures," Nano Lett. 2017. 